\title{
Optimal Control of Gearshift in Automatic Mechanical Transmission
}

\author{
Liying Miao1, Xiusheng Cheng1, Zhonghua Liu2, Xuesong Li1 \& Xi Liu3 \\ 1 State Key Laboratory of Automotive Simulation and Control, Jilin University, Changchu, China \\ 2 China FAW Co., Ltd R\&D Center, Changchun, China \\ 3 SAIC motor passenger vehicle company, Shanghai, China
}

Keywords: AMT, wet clutch, pressure control, fuzzy control.

\begin{abstract}
In order to solve the problems existed in gearshift process of automatic mechanical transmission(AMT), the control strategy base on fuzzy control was presented with regarding of engine running state and clutch engaging state. The wet clutch target pressure of shifting process was determined by the fuzzy controller. According to the error of target pressure and actual pressure, a wet clutch pressure intelligent control arithmetic base on Radial Basis Function Neural Network(RBFNN) was designed to realize accuracy control of clutch pressure. The vehicle gear shifting experiment was conducted to verify the control strategy.
\end{abstract}

\section{Introduction}

AMT is based on original manual transmission, it has the advantages of high transmission efficiency and low manufacturing cost compared with traditional hydrodynamic mechanical automatic transmission(AT). But power interruption existed during shifting, and shifting comfort is less than AT. Therefore, clutch control during shifting process was the core point and difficulty of AMT. It not only improves smooth and prevents excessive jerk and ensures the stable operation of the engine, also ensures that the friction is not too large. If the clutch engage too fast, will generate shifting jerk, on the contrary, the shifting time is too long, friction of clutch is violent, temperature of clutch oil rise, will affect the service life of the clutch.

On the analysis of shifting process, the writer improved the control strategy, designed a fuzzy controller to calculate the target pressure of clutch, through the real-time monitoring of the clutch pressure increment. The writer also make the design base on RBFNN, a wet clutch pressure intelligent control system to achieve a precise control of the clutch pressure, and the results were verified by experiment.

\section{Clutch control strategy of shifting process}

Wet clutch went through three kinds of state during shifting process: apart state, friction state, engaging state. In friction state, clutch engaged partly, not entirely, and transmitted friction torque. Therefore, the driving and the driven plates of the wet clutch and clutch oil temperature had grate effect on wet clutch dynamic friction coefficient.

A fuzzy control system was designed, which use the rotation speed difference between the driving and the driven plates of the wet clutch, clutch oil temperature, engine speed as input parameters, also changing rate of clutch pressure as output parameter[1, 2]. However, in order to prevent engine flameout or shake, engine speed must be monitored in real time.

The control rules of fuzzy controller were fuzzy conditional sentence which was composed of several linguistic variables. According to the need of fuzzy pressure controller, select the input and output variables respectively as: the rotation speed difference between the driving and the driven plates of the wet clutch $\Delta \omega$ "very small” (VS ) ,"small” ( S ) ,"middle” (M) ,"big” (B) ,"very big”

(VB); clutch oil temperature $t_{\text {tem }}$ : "very small” (VS), "small” ( S ), “middle” (M), "big” (B), "very big” (VB) ; engine speed $\omega_{e}$ : “very small” (VS ) ,"small” (S) ,“middle” (M) ,"big” (B) ,"very big”( VB ); changing rate of clutch pressure $\Delta p$ : "very small”( VS ), “small”( $\mathrm{S}$ ), "middle”( $\mathrm{M}$ ), "big” 
(B) ,"very big” (VB) .

The membership function of each fuzzy variable was triangular distribution. Each fuzzy subset membership curve was shown in fig.1.

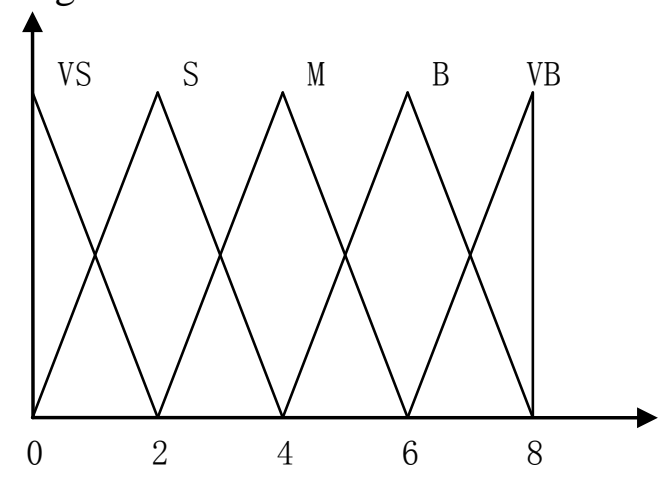

Figure 1: Membership function

Because fuzzy controller adopted a variety of input variables, in order to avoid large storage capacity and difficulty in adjustment, the writer designed the structure of two layers with multiple rules[3,11]. The schematic of fuzzy control was shown in fig.2. Fuzzy controller had two layers of inference, the first layer, made an inference from the rotation speed difference between the driving and the driven plates of the wet clutch $\Delta \omega$ and clutch oil temperature $t_{\text {tem }}$, got clutch dynamic friction coefficient $\mu$, the second layer, made an inference from clutch dynamic friction coefficient $\mu$ and engine speed $\omega_{e}$, got changing rate of clutch pressure $\Delta p$.

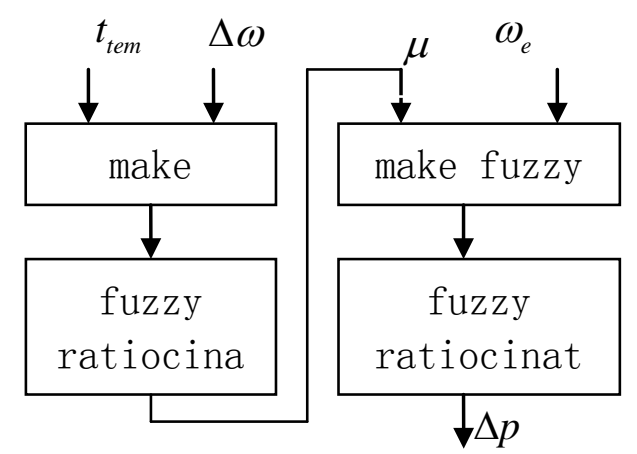

Figure 2: Schematic diagram of fuzzy control

Tab. 1 is the fuzzy rule table of dynamic friction coefficient $\mu$. Tab.2 is the fuzzy rule table of changing rate of clutch pressure $\Delta p$.

\begin{tabular}{|c|c|c|c|c|c|}
\hline \multirow{2}{*}{$\Delta \omega$} & \multicolumn{5}{|c|}{$t_{\text {tem }}$} \\
\cline { 2 - 6 } & VS & S & M & B & VB \\
\hline VS & VS & S & M & B & VB \\
\hline S & S & M & B & M & S \\
\hline M & M & B & M & B & M \\
\hline B & S & M & B & M & S \\
\hline VB & VS & S & M & S & VS \\
\hline
\end{tabular}

Table 1: Fuzzy rule table of $\mu$ 


\begin{tabular}{|c|c|c|c|c|c|}
\hline \multirow[t]{2}{*}{$\omega_{e}$} & \multicolumn{5}{|c|}{$\mu$} \\
\hline & VS & $\mathrm{S}$ & $\mathrm{M}$ & B & VB \\
\hline VS & VS & $S$ & $\mathrm{~S}$ & VS & VS \\
\hline $\mathrm{S}$ & $\mathrm{M}$ & $\mathrm{M}$ & $\mathrm{S}$ & $\mathrm{S}$ & $\mathrm{S}$ \\
\hline $\mathrm{M}$ & B & B & $\mathrm{M}$ & $\mathrm{M}$ & $\mathrm{M}$ \\
\hline B & B & B & $\mathrm{M}$ & $\mathrm{M}$ & $\mathrm{S}$ \\
\hline VB & VS & VB & B & B & $\mathrm{M}$ \\
\hline
\end{tabular}

Table 2: Fuzzy rule table of $\Delta p$

The paper used Mamdani direct reasoning method and "max-min" method to do synthetic operation of fuzzy relation, and used gravity method for defuzzification, obtained the dynamic friction coefficient $\mu$ l, as shown in fig.3.

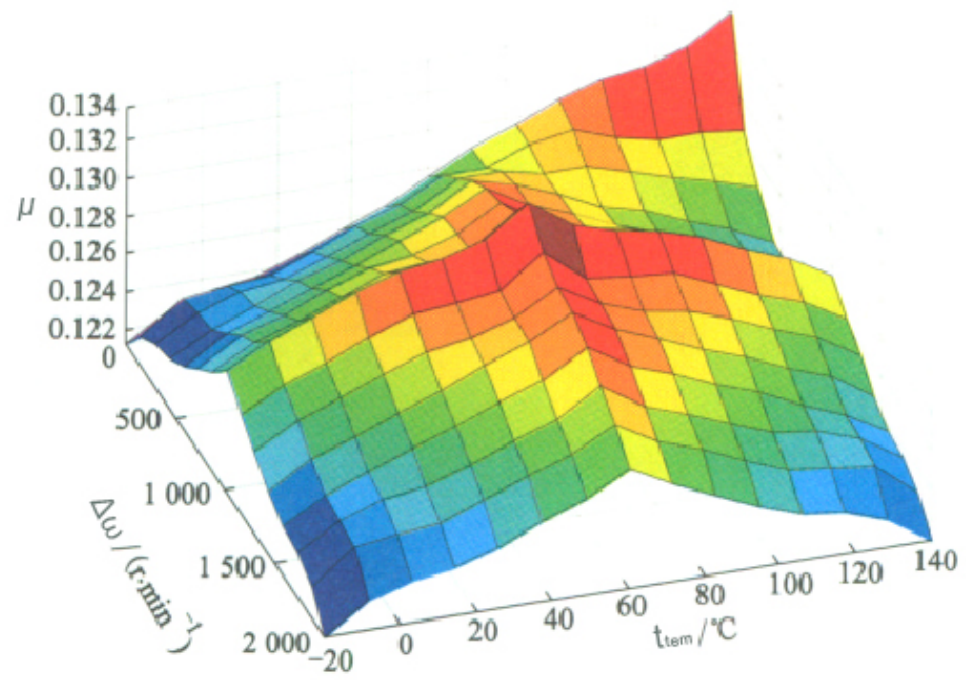

Figure 3:Fuzzy rule surface of $\mu$

\section{Evaluation index of shifting quality}

Jerk and friction were the main evaluation index of shifting performance. Jerk is defined as the changing rate of longitudinal acceleration in unit time, usually use $j=\frac{d^{2} v}{d t^{2}}, v$ was vehicle speed. From $v=\frac{\omega r}{i_{g} i_{0}}$, the mathematical expressions for $j$ is:

$$
j=\frac{d^{2} v}{d t^{2}}=\frac{r}{i_{g} i_{0}} \frac{d^{2} \omega}{d t^{2}}=\frac{1}{\delta m} \frac{i_{g} i_{0} \eta}{r} \frac{d T_{c}}{d t}
$$

In this formula, $r$ represents wheel radius, $i_{g}$ represents main reduction ratio, $i_{0}$ represents,

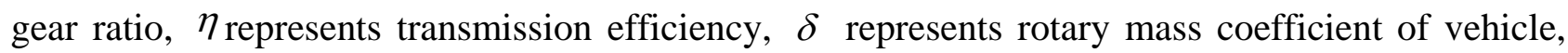
$m$ represents quality of vehicles, $T_{c}$ represents clutch torque.

Friction is sliding friction work between the driving and the driven plates of the wet clutch, the mathematical expressions is:

$$
L=\int_{t_{0}}^{t_{1}} T_{c}\left(\omega_{e}-\omega_{c}\right) d t
$$

In this formula, $t_{0}$ represents the time when clutch engaging and starting transfer torque, $t_{1}$ represents the time rotation speed between the driving and the driven plates of the wet clutch achieve synchronization.

Friction had relationship with clutch oil temperature in wet clutch, when friction was large, oil 
temperature rose fast. Friction was represented by oil temperature in the paper. Jerk was related to clutch engaging speed by eqn(1). Friction was related to rotation speed difference between the driving and the driven plates of the wet clutch, clutch torque and friction time by eqn(2). Therefore, jerk and friction were contradictory as evaluation index[4,5].

In wet clutch, clutch temperature has the close relationship with the friction, when the friction become too large, oil temperature will increases rapidly, so the paper use the clutch oil temperature to reflect friction. During the shifting process, how to balance the jerk and clutch oil temperature, is the core point of the control strategy

\section{Optimal control of clutch pressure during shifting process}

The clutch torque is related to many factors, which is a nonlinear complex model. Secondly, after long time using, clutch model will be distortion and clutch torque will certainly change, even if the initial phase of the clutch model is accurate[6,7,8]. Solenoid valve also has the same problems after long using, the accuracy of the model will be decreased. Meanwhile, the machining precision of each part with different batches of production can not be completely the same, there will be certain errors[9,10]. In conclusion, the writer used RBFNN adaptive controller to compensate for the clutch and solenoid valve model. According to the error between the clutch target pressure and actual pressure value, the controller generated control current to amend, to improve the control accuracy of clutch pressure, and to compensate model distortion automatically.

The RBFNN simulation model was established using Simulink, as shown in fig.4. Solenoid value module was the simulation module of clutch pressure control valve, RBFNN module was the simulation model of RBFNN, Signal was signal generation module of the model. $p_{t}$ was the clutch target pressure, $p_{r}$ was the clutch actual pressure, $i$ was the control current. The target pressure $p_{t}$ and the actual pressure $p_{r}$ were the input of RBFNN, output was the control current $i$. Solenoid value module calculated the actual clutch pressure according to the input control current $i$. The simulation curve was shown in the fig.5, it had high precision in following and small control error.

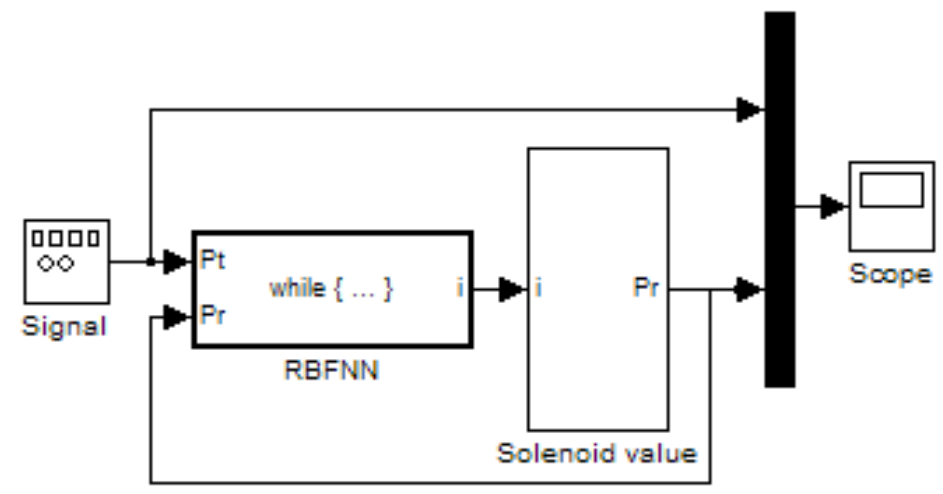

Figure 4: RBFNN simulation model

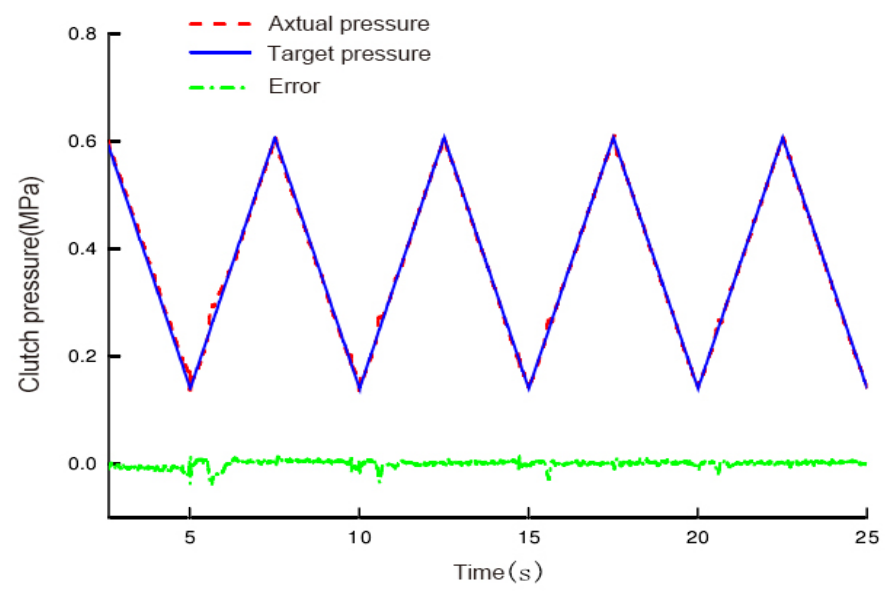


Figure 5: Simulation curve of pressure

\section{Experiment results and analysis}

The controller of the test vehicle used IFINEON C164CI microprocessor as the main control chip, analog signal used RC filter circuit for signal filtering, pulse signal used photoelectric isolation chip to do isolation processing, the data collected through the CAN communication interface to upload to the computer.

Fig. 6 to fig. 8 was the experiment result. As shown in fig.6, engine speed was pulled down slowly, the shifting process lasted about 1 second, the shifting action was quickly. As shown in fig.7 vehicle speed was changing stably, the throttle was stable at $10 \%$, the maximum of jerk was not more than $9 \mathrm{~m} / \mathrm{s}^{3}$. As shown in fig.8, after shifting several times, oil temperature was stable at about $70^{\circ} \mathrm{C}$, the maximum was not more than $80^{\circ} \mathrm{C}$. The results show that shifting control strategy meets the requirements of smooth and rapid ride in gear shift process.

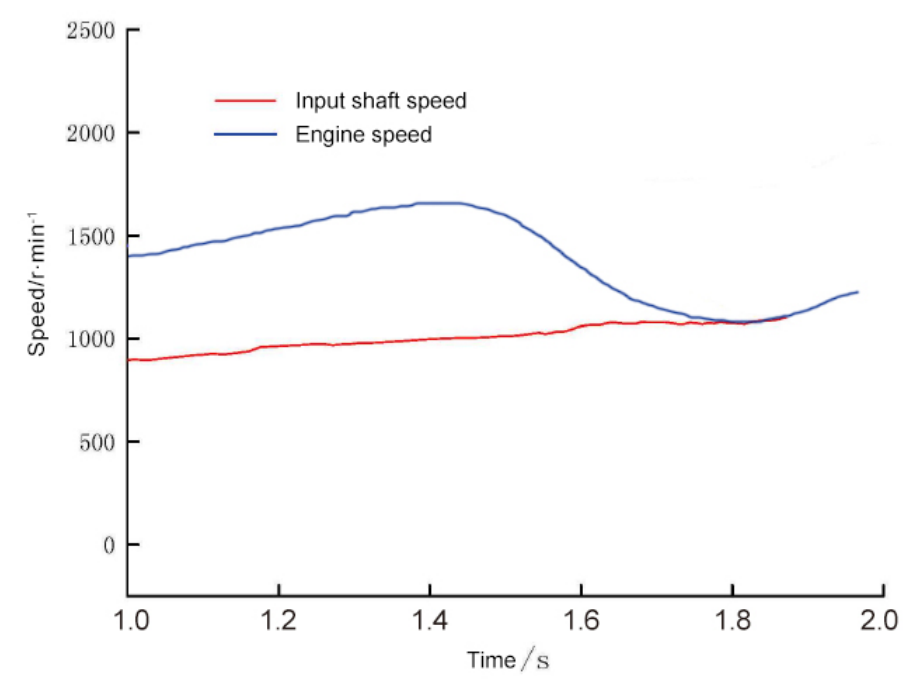

Figure 6: The engine speed, input shaft speed in 1-2 upshift

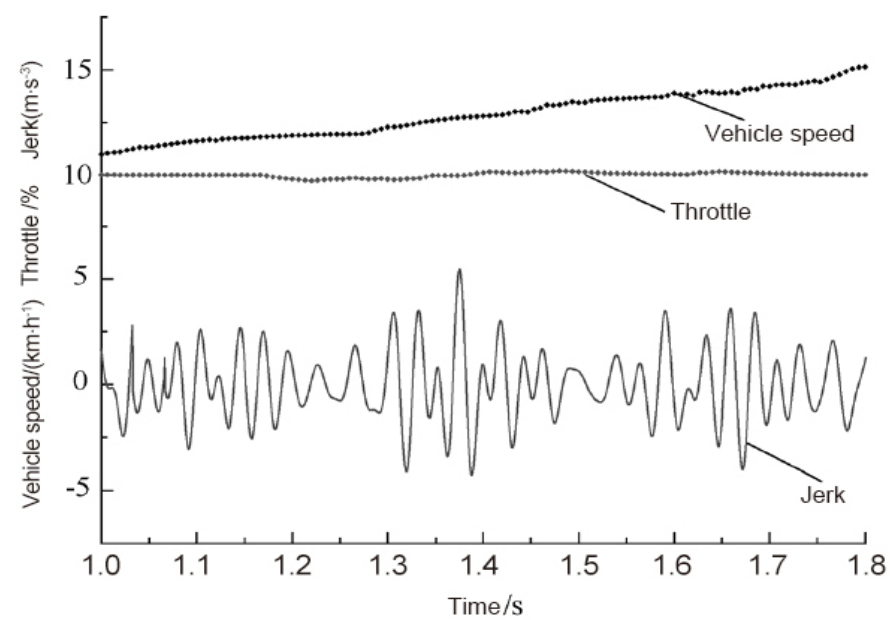

Figure 7: The vehicle speed, throttle, jerk in 1-2 upshift 


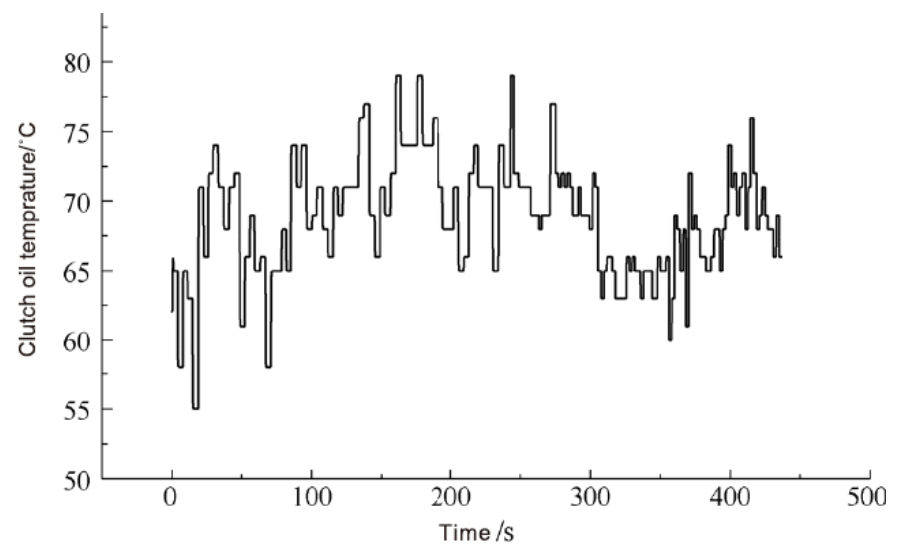

Figure 8: Oil temperature during multiple continuous gearshifts

\section{Conclusion}

On the analysis of shifting process of AMT with wet clutch, fuzzy control was used to calculate the target pressure of clutch. RBFNN adaptive controller was established to realize accuracy control of clutch pressure. The optimal control meet the requirements of shifting process, provided a reference control method for problems of shifting.

\section{Acknowledgment}

This work is supported by the National Nature Science Foundation of China (51305156).

\section{References}

[1]Liu Xi, Cheng Xiusheng, Feng Wei. Optimal control of gear shift in wet dual-clutch automatic transmission, Transactions of the CSAE, 27(6): pp. 152-156, 2011.

[2]Zhang Zhi-gang, Study on Several Working Characteristics of Wet Clutch, Zhejiang University, pp. 68-70, 2010.

[3] Feng Wei, Study on Starting and Shifting Control of Wet Double Clutch Transmission, Jilin University, pp. 40-61, 2010.

[4] Yinshu Wang, Xiusheng Cheng, Wei Feng, The Wet Clutch Pressure Control of Dual Clutch Transmission Based on FCMAC, edited by Applied Mechanics and Materials, v157-158, pp. 76-84.

[5] Cheng Yu, Research on Control Technology for Torque Assist Mechanism AMT Base on the Wet Clutch, Jilin University, pp. 105-112, 2014.

[6] J.Kim and S.Choi, Self-energizing Clutch Actuator System, FISTIA 2010 Congress, Budapest.

[7].Sun Dongye, Hu Fengbing. Simulation and test on warpage characteristics of wet multi plate clutch, Journal of Chongqing University, 33(5):pp.1-6, 2010.

[8].Han Yong. Simulate and analysis on wet friction plates based on CFD technology. Jilin:Jilin University, pp. 35-39, 2011.

[9]. Zhu Hongqing. Study on heat and friction characteristics of wet clutch, Zhejiang University, pp. 67-72, 2012.

[10].Zhang jinle, Ma Biao. Simulation on thermal characteristics of wet shifting clutch, Journal of Jilin University, 41(2):pp.321-326, 2011.

[11].Chen Yaofei. Study on the characteristics of wet multi plate clutch and the friction heat failure. Chongqing University, pp. 86-92, 2009. 